\title{
Determinants of Functional Outcomes Using Clinical Pathways for Rehabilitation After Hip Fracture Surgery
}

\author{
Ji-Ho Kang ${ }^{1}$, Gangpyo Lee ${ }^{2}$ Kyoung-Eun Kim³ , Young-Kyun Lee ${ }^{4}$, Jae-Young Lim ${ }^{1}$ \\ ${ }^{1}$ Department of Rehabilitation Medicine, Seoul National University College of Medicine, Seoul National University Bundang Hospital, Seongnam, \\ ${ }^{2}$ Department of Rehabilitation Medicine, Incheon Workers' Compensation Hospital, Incheon, ${ }^{3}$ Department of Rehabilitation Medicine, The \\ Armed Forces Capital Hospital, Seongnam, ${ }^{4}$ Department of Orthopedic Surgery, Seoul National University College of Medicine, Seoul National \\ University Bundang Hospital, Seongnam, Korea
}

Corresponding Author: Jae-Young Lim, MD, PhD Department of Rehabilitation Medicine, Seoul National University College of Medicine, Seoul National University Bundang Hospital, 82 Gumi-ro 173beon-gil, Bundang-gu, Seongnam 13620, Korea

Tel: $+82-31-787-7739$

Fax: +82-31-787-4051

E-mail:drlim1@snu.ac.kr

\begin{abstract}
Background: This study evaluated functional outcomes using newly established clinical pathways after hip fracture surgery in older adults and analyzed the major determinants of successful functional outcomes in rehabilitation programs using standardized clinical pathways. Methods: This was a retrospective cohort study performed in a tertiary rehabilitation facility. A total of 220 patients who had received unilateral hip fracture surgery were followed up from immediately after surgery to 6 months postoperatively. Clinical pathways for rehabilitation included early, individualized rehabilitation, education for activities of daily living, review of general medical conditions, and arrangement of discharge settings. One rehabilitation specialist consecutively checked ambulatory function using 3-level grading, and patients were classified into good recovery and poor recovery groups based on ambulatory function at 6 months postoperatively. Logistic regression analysis was performed using 7 representative variables (age, sex, bone mineral density, Mini-Mental Status Examination [MMSE], Berg Balance Scale [BBS], premorbid ambulatory function, and length of hospital stay). Results: A total of $86.8 \%$ of patients could walk with or without assistance at 6 months after surgery and $75.5 \%$ of patients involved in the rehabilitation program were classified into the good recovery group in this study. The good recovery group showed higher MMSE and BBS scores compared with the poor recovery group. The factors in the model most strongly correlated with recovery were MMSE and BBS. Conclusion: This study showed that a well-designed rehabilitation program could improve ambulatory function in older patients after hip fracture surgery and that cognitive impairment and poor balance control may inhibit the recovery of ambulatory function.
\end{abstract}

Key Words: Hip fracture, Clinical pathway, Rehabilitation program, Ambulatory function, Functional recovery

\section{INTRODUCTION}

Hip fracture is a geriatric disease with multiple predisposing factors that may lead to falls, such as osteoporosis, weakness, and dizziness. The incidence of hip fracture differs by country. In South Korea, the age-standardized annual incidence rate of hip fractures in 2003 was 104.06 per 100,000, including 146.38 per 100,000 for women and 61.72 per 100,000 for men. ${ }^{1,2)}$ Older people have a 5 - to 8 -fold increased risk of mortality during the first 3 months after hip fracture, and increased annual mortality persisted over time in both women and men following hip fracture. ${ }^{3)}$ Likewise, hip fractures, which mostly result from falls related to multiple predisposing risk factors in geriatric populations, are a major public health problem.

To achieve ambulatory function after surgical treatment, multidisciplinary rehabilitation has been emphasized by geriatric and inpatient rehabilitation units. Multidisciplinary rehabilitation for hip fractures includes early mobility and self-care training, postoperative management monitored by a geriatrician, high-frequency additional occupational therapy combined with physical therapy, and accelerated discharge. Furthermore, home-based rehabilitation is required to decrease complications, reduce transfers to intensive care units or nursing homes, and improve walking ability. Halbert et al., ${ }^{4)}$ in their review of randomized trials, reported that multidisciplinary rehabilitation decreased the likelihood that patients with hip fracture would have a poor outcome, including death or admission to a nursing home, by an additional 16\% compared 
with traditional rehabilitation. Recently, effective clinical pathways have been introduced in rehabilitation programs for many types of geriatric disease. ${ }^{5-7)}$ Many studies have explored the effects of postoperative rehabilitation in hip fracture, and several clinical pathways for postoperative rehabilitation of hip fracture have been proposed. ${ }^{7-11)}$ However, clinical pathways for the rehabilitation of acute hip fracture in Korea have not been well established due to the limited integration of care among orthopedic, geriatric, and rehabilitation specialties. Therefore, specialized inpatient rehabilitation services are not properly provided for patients with acute hip fracture in most general hospitals. For this reason, we developed clinical pathways for the rehabilitation of acute hip fracture, and patients with acute hip fracture have been involved since the program's inception.

The purpose of the study was to evaluate the functional outcomes of newly established clinical pathways after hip fracture surgery in older adults, and to analyze the major determinants of successful functional outcomes using standardized clinical pathways.

\section{MATERIALS AND METHODS}

\section{Subjects}

This was a retrospective cohort study performed in a tertiary rehabilitation facility. Between November 2009 and December 2015, 883 patients who had received fracture surgery and were transferred to the Department of Rehabilitation Medicine, and who agreed to be enrolled in the study were registered. We recruited 220 patients who met the following inclusion criteria: (1) hospitalization period between November 2009 and December 2015; (2) duration of inpatient rehabilitation $>1$ week; (3) age $\geq 65$ years; (4) acute unilateral hip fracture (femur neck, intertrochanteric, subtrochanteric); and (5) time interval between onset of injury and operation $\leq 2$ weeks. Surgical treatments included bipolar hemiarthroplasty, total hip arthroplasty, and open reduction and internal fixation (Fig. 1). Patients who had hip surgery due to osteoarthritis, infected hip, or avascular necrosis, and those who were readmitted due to periprosthetic fracture or prosthetic loosening were excluded. After patients were transferred to the Department of Rehabilitation Medicine, they participated in the Rehabilitation program for Hip fracture Functional Outcome Study (ReHipFOS) (Fig. 1).

\section{Clinical Rehabilitation Pathway}

ReHipFOS, the clinical pathway for rehabilitation evaluated in this study, includes early individualized rehabilitation, education for activities of daily living (ADLs), review of general medical conditions, appropriate management, establishment of further plans, and arrangement for discharge settings
(Fig. 2). The rehabilitation and education elements of this clinical pathway included transfer and gait training with an assistive device, education about hip precautions in ADLs, functional training for independent $A D L$, strengthening exercises for the hip girdle muscles, stretching exercises to increase flexibility of the lower extremities including the hip muscles, and fall-prevention education. Specifically, patients were involved in physical therapy twice a day and occupational therapy once a day for at least 20 minutes per session during the hospital day. Patients who were unable to walk before surgery received physical therapy on the tilt table, standing frame, and parallel bars, and patients who were able to walk before surgery employed increased weight-bearing according to the type of surgery and began walking with assistive devices at an early stage. When ambulatory function improved, increased gait endurance was encouraged and lower extremity strengthening exercises were performed using isotonic exercise equipment and elastic bands. Patients were also trained using balance equipment. The study protocol was approved by the Institutional Review Board of Seoul National University Bundang Hospital (approval number: B-1101-119-110).

\section{Functional Evaluation}

To evaluate ambulatory function, one geriatric rehabilitation specialist consecutively assessed patient ambulatory function during the premorbid stage, after transfer to rehabilitation medicine, at discharge, and at the 6-month follow-up using 3-level grading of ambulatory function modified from the Functional Ambulation Category or Classification, as fol-

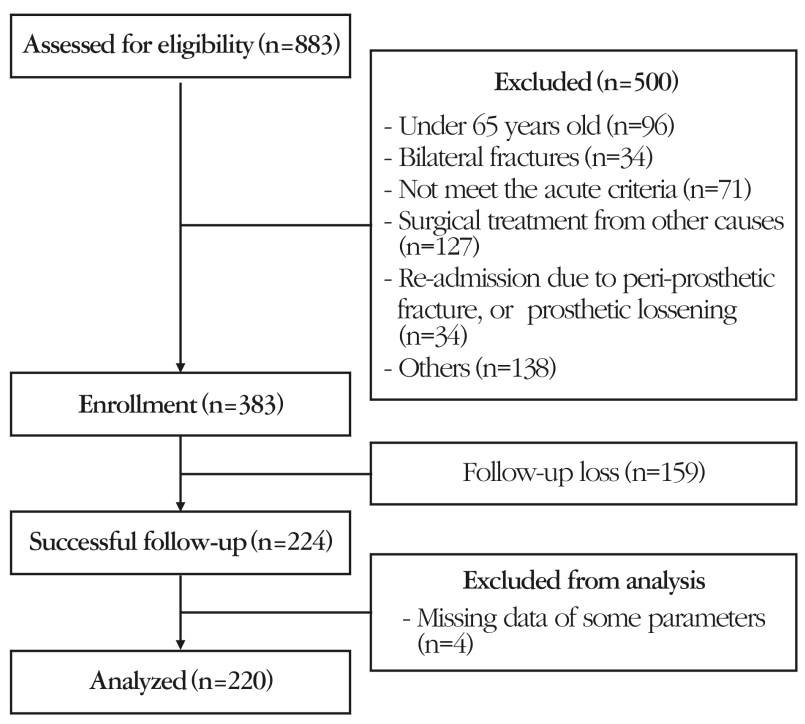

Fig. 1. Flow chart of patient inclusion, with reason for exclusion and total study population. 
lows. Level 3: Ambulation is independent and without supervision or physical assistance from another person. Except for parallel bars, the patient may use assistive devices, orthoses, and prostheses. Level 2: The individual is able to walk at least 10 feet outside the parallel bars with physical assistance from only one person. Except for parallel bars, mechanical assistance from any device or ambulation aid may be used. Level 1: Individual ambulatory function does not advance. Comorbidity status, dual-energy X-ray absorptiometry for bone densitometry, and length of hospital stay were recorded after transfer to rehabilitation medicine and at discharge. In addition, data for the following scales were used to evaluate functional and cognitive status.

\section{1) Modified Barthel Index (MBI)}

The MBI measures individual performance for 10 ADLs. ${ }^{12)}$ The scores for each item in the MBI are based on the amount of physical assistance required to perform the task, and the items are summed to give a score ranging from 0 to 100 .

\section{2) Mini-Mental Status Examination (MMSE)}

This screening test is a brief, objective measure of cognitive functioning. ${ }^{13)}$ The MMSE has a maximum score of 30 points, and the questions are grouped into 7 categories, each representing a different cognitive domain or function.

\section{3) Geriatric Depression Scale (GDS)}

The GDS is the most commonly used depression self-report scale and consists of 30 items. ${ }^{14)}$ The items, which have yes or no answers, have been useful in distinguishing depressed from normal subjects based on characteristics of depression in older people.

\section{4) The 10-m walk test}

This test is a simple gait assessment that can be used to determine walking speed. For the test, the time taken to walk $10 \mathrm{~m}$ is measured using a stopwatch, and walking speed is calculated by dividing the distance covered by the time (m/sec).

\section{5) Berg Balance Scale (BBS)}

The BBS was developed as a performance-oriented measure of balance in older adults. ${ }^{15)}$ The items include simple mobility tasks and more difficult tasks. The BBS consists of 14 items scored on a scale of 0 to 4 ; the maximum total score is 56 .

\section{Data Analysis}

We compared ambulatory function during the premorbid

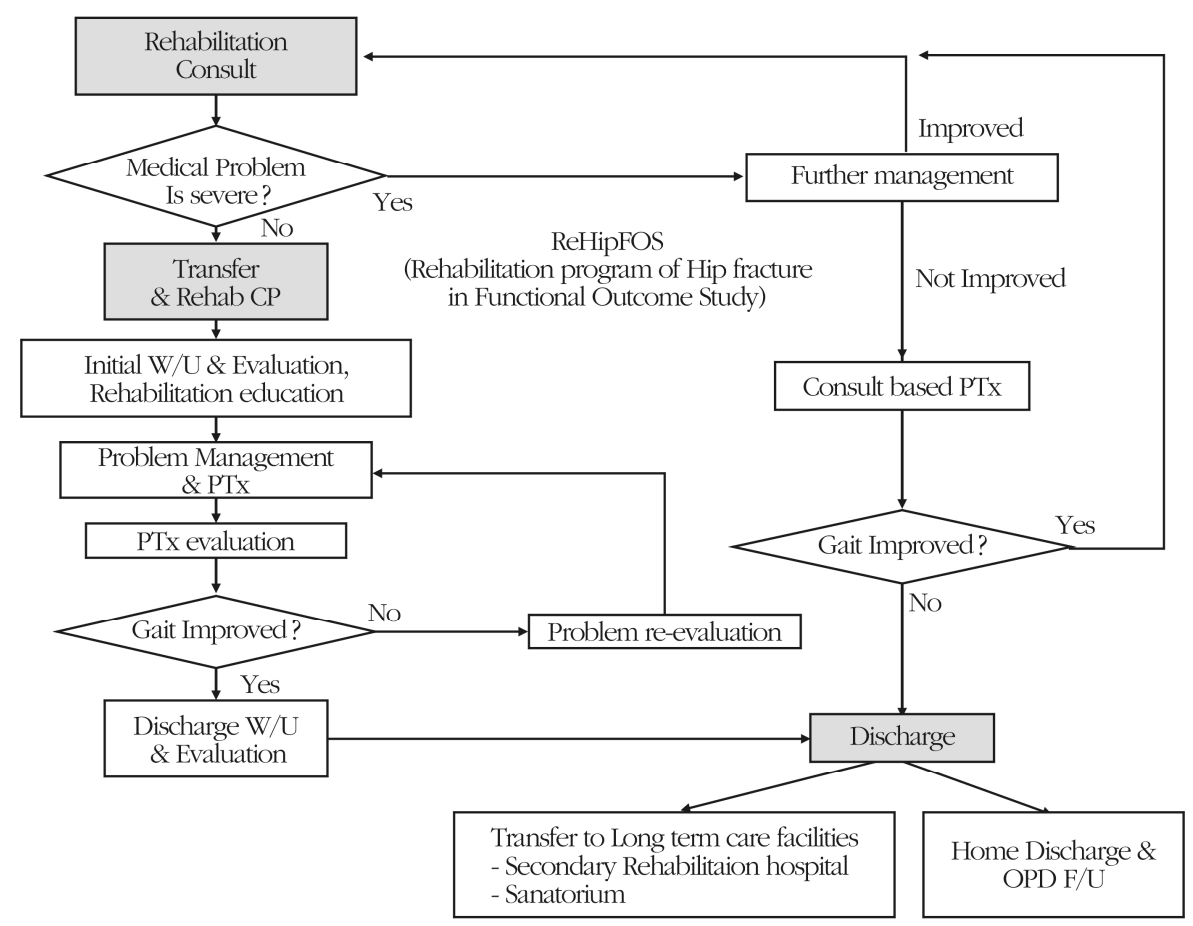

Fig. 2. Flow chart of subject recruitment. CP, clinical pathway; PTx, physical therapy; W/U, work up; OPD, outpatient department; F/U, follow-up. 
state and at 6 months after surgery and classified patients into 2 groups accordingly. Patients whose ambulatory func-

Table 1. Patient demographics $(n=220)$

\begin{tabular}{|c|c|}
\hline Variable & Value \\
\hline Age (yr) & $80.6 \pm 7.4$ \\
\hline \multicolumn{2}{|l|}{ Sex } \\
\hline Male & 61 \\
\hline Female & 159 \\
\hline \multicolumn{2}{|l|}{ Laterality } \\
\hline Right & 108 \\
\hline Left & 112 \\
\hline Body mass index $\left(\mathrm{kg} / \mathrm{m}^{2}\right)$ & $21.4 \pm 3.9$ \\
\hline BMD (T-score) & $-3.1 \pm 1.2$ \\
\hline Osteoporosis & 175 \\
\hline Osteopenia & 45 \\
\hline MMSE & $19.4 \pm 7.2$ \\
\hline MBI & $33.1 \pm 20.1$ \\
\hline BBS & $18.7 \pm 15.3$ \\
\hline GDS & $11.2 \pm 7.6$ \\
\hline Time taken to walk $10 \mathrm{~m}(\mathrm{sec})$ & $59.9 \pm 52.5$ \\
\hline Previous hip surgery & 27 \\
\hline \multicolumn{2}{|l|}{ Level of fracture } \\
\hline Intertrochanteric & 114 \\
\hline Femur neck & 102 \\
\hline Subtrochanteric & 4 \\
\hline \multicolumn{2}{|l|}{ Type of operation } \\
\hline Bipolar hemiarthroplasty & 157 \\
\hline Total hip replacement arthroplasty & 7 \\
\hline Open reduction and internal fixation & 56 \\
\hline \multicolumn{2}{|l|}{ Time interval (day) } \\
\hline Onset of injury to operation & $5.6 \pm 7.3$ \\
\hline Operation to start of physical therapy & $7.8 \pm 6.1$ \\
\hline Admission to discharge & $27.8 \pm 36.0$ \\
\hline \multicolumn{2}{|l|}{ Discharge setting } \\
\hline Home & 109 \\
\hline Secondary rehabilitation hospital & 95 \\
\hline Nursing home & 16 \\
\hline
\end{tabular}

Values are presented as the mean \pm standard deviation or number. BMD, bone mineral density; MMSE, Mini-Mental State Examination; CDR, Clinical Dementia Rating; MBI, Modified Barthel Index; BBS, Berg Balance Scale; GDS, Geriatric Depression Scale. tion improved or had not deteriorated after surgery were classified into the good recovery group, and the others, including patients whose ambulatory function was at level 1 before and after surgery, were classified into the poor recovery group. We used the Mann-Whitney test, Kruskal-Wallis test, and chi-square test to compare groups. Multiple logistic regression analysis was used to identify variables that had significant effects on ambulatory function.

\section{RESULTS}

\section{Demographic Data}

The demographic characteristics of patients are listed in Table 1. Of the 220 patients (mean age, $80.6 \pm 7.4$ years; 61 male and 159 female patients), 108 had fractures on their right side, and 112 on the left. A total of 114 patients had previous hip surgery. The mean body mass index (BMI) was 21.4 \pm 3.9 , and mean T-score of bone mineral density was $-3.1 \pm 1.2$. With regard to cognitive functioning, the mean MMSE score was 19.4 \pm 7.2 . The mean BBS score was $18.7 \pm 15.3$, and the mean time taken to walk $10 \mathrm{~m}$ was $59.9 \pm 52.5$ seconds. In addition, the mean GDS score was 11.2 \pm 7.6 . The patients were classified into 3 groups based on each of the following characteristics: level of fracture (114 intertrochanteric; 102 femur neck; 4 subtrochanteric); type of operation (157 bipolar hemiarthroplasty; 7 total hip replacement arthroplasty; 56 open reduction and internal fixation); and discharge setting (109 home; 95 secondary rehabilitation hospital; 16 nursing home).

\section{Recovery of Ambulatory Function After Hip Fracture}

Ambulatory function at each time point is listed in Table 2. Although $93.6 \%$ of patients could walk with or without assistance (independent ambulation: 81.8\%; assisted ambulation: $11.8 \%$ ) in the premorbid state, only $72.3 \%$ were able to walk (independent ambulation: 1.8\%; assisted ambulation: $70.5 \%$ ) when they were transferred to rehabilitation medicine. A total of $85.5 \%$ of patients could walk with or without assistance (independent ambulation: 35.0\%; assisted ambulation: $50.5 \%$ ) at discharge, and $86.8 \%$ were able to walk (independent ambulation: 70.9\%; assisted ambulation: 15.9\%) at 6 months after surgery. Premorbid ambulatory function,

Table 2. Recovery of ambulatory function after hip fracture surgery

\begin{tabular}{lccrr}
\hline \hline & Premorbid & Transfer & Discharge & 6-Month follow-up \\
\hline Independent ambulation (3) & $180(81.8)$ & $4(1.8)$ & $77(35.0)$ & $156(70.9)$ \\
Assisted ambulation (2) & $26(11.8)$ & $155(70.5)$ & $111(50.5)$ & $35(15.9)$ \\
Nonambulatory (1) & $14(6.4)$ & $61(27.7)$ & $32(14.5)$ & $29(13.2)$ \\
\hline
\end{tabular}

Values are presented as number (\%). 
the type of fracture, and the type of surgery did not have significant effects on the prognosis for ambulatory function after hip fracture.

\section{Comparison Between Premorbid and 6-Month Postoperative Ambulatory Function}

Of the 220 patients, 166 were in the good recovery group, and 54 were in the poor recovery group (Fig. 3). Of 180 patients whose ambulatory function was level 3 before surgery, 136 $(75.6 \%)$ remained at level 3 after surgery. Of 26 patients whose ambulatory function was at level 2 before surgery, $21(80.8 \%)$ improved from level 2 to 3 or remained at level 2. In addition, of the 14 patients whose ambulatory function was level 1 before surgery, $9(64.3 \%)$ improved to level 2 or 3 , i.e., good recovery.

Of the 180 patients whose ambulatory function was level 3 before surgery, ambulatory function deteriorated to level 1 or 2 in $44(24.4 \%)$ after surgery. Of the 26 patients whose ambulatory function was level 2 before surgery, ambulatory function in $5(19.2 \%)$. decreased to level 1. Five patients whose ambulatory function was at level 1 before and after surgery were in the poor recovery group.

The demographic characteristics of each group are listed in Table 3. There was no significant difference in the pro-

\begin{tabular}{|c|c|c|c|c|}
\hline \multicolumn{5}{|c|}{ Good recovery $75.5 \%(166 / 220)$} \\
\hline Premorbid & PDD 6 mo & & $\mathrm{N}$ & $\%$ \\
\hline & & $3 \rightarrow 3$ & $136 / 180$ & 75.6 \\
\hline & & $2 \rightarrow 3$ & $15 / 26$ & \\
\hline & & $2 \rightarrow 2$ & $6 / 26$ & \\
\hline & & $1 \rightarrow 3$ & $5 / 14$ & \\
\hline & & $1 \rightarrow 2$ & $4 / 14$ & \\
\hline
\end{tabular}

\begin{tabular}{|l|l|l|l|}
\hline \multicolumn{5}{|c|}{ Poor prognosis 24.5\% (54/220) } \\
\hline Premorbid & \multicolumn{1}{|c|}{ PDD 6 mo } \\
\cline { 3 - 4 }
\end{tabular}

Fig. 3. (A). Premorbid and 6-month follow-up ambulatory function in the good recovery group. (B) Premorbid and 6-month follow-up ambulatory function in the poor recovery group. POD, postoperative day. portion of those with poor recovery according to sex, laterality, BMI, etc. The good recovery group showed higher MMSE and BBS, but lower CDR than the poor recovery group. There were no significant differences between the 2 groups except in MMSE, BBS, and CDR.

\section{Factors Influencing Ambulatory Function at the 6-Month Follow-up}

The results of stepwise multiple regression analysis for am-

Table 3. Differences between the good recovery and poor recovery groups

\begin{tabular}{|c|c|c|}
\hline Variable & $\begin{array}{c}\text { Good } \\
\text { recovery } \\
(\mathrm{n}=166)\end{array}$ & $\begin{array}{c}\text { Poor } \\
\text { recovery } \\
(\mathrm{n}=54)\end{array}$ \\
\hline Age (yr) & $80.2 \pm 7.4$ & $81.9 \pm 7.2$ \\
\hline \multicolumn{3}{|l|}{ Sex } \\
\hline Male & $46(27.7)$ & $15(27.8)$ \\
\hline Female & $120(72.3)$ & $39(72.2)$ \\
\hline \multicolumn{3}{|l|}{ Laterality } \\
\hline Right & $83(50.0)$ & $25(46.3)$ \\
\hline Left & $83(50.0)$ & $29(53.7)$ \\
\hline Body mass index $\left(\mathrm{kg} / \mathrm{m}^{2}\right)$ & $21.2 \pm 3.8$ & $21.7 \pm 4.4$ \\
\hline T-score of bone mineral density & $-3.1 \pm 1.2$ & $-3.0 \pm 1.2$ \\
\hline MMSE & $20.6 \pm 6.7^{* * *}$ & $15.5 \pm 7.1$ \\
\hline MBI & $35.2 \pm 19.7$ & $26.5 \pm 19.6$ \\
\hline BBS & $21.4 \pm 15.3^{* * *}$ & $10.9 \pm 12.1$ \\
\hline GDS & $10.6 \pm 7.3$ & $13.4 \pm 8.2$ \\
\hline 10 -m walk test (sec) & $58.5 \pm 44.7$ & $64.6 \pm 72.7$ \\
\hline Previous hip surgery & 21 & 6 \\
\hline \multicolumn{3}{|l|}{ Level of fracture } \\
\hline Intertrochanteric & $89(53.7)$ & $25(46.3)$ \\
\hline Femur neck & $73(43.9)$ & $29(53.7)$ \\
\hline Subtrochanteric & $4(2.4)$ & 0 \\
\hline \multicolumn{3}{|l|}{ Type of operation } \\
\hline Bipolar hemiarthroplasty & $117(70.5)$ & $40(74.1)$ \\
\hline Total hip replacement arthroplasty & $6(3.6)$ & $1(1.9)$ \\
\hline Open reduction and internal fixation & $43(25.9)$ & $13(24.0)$ \\
\hline \multicolumn{3}{|l|}{ Time interval (day) } \\
\hline Onset of injury to operation & $5.4 \pm 8.0$ & $6.3 \pm 4.3$ \\
\hline Operation to start of physical therapy & $7.6 \pm 5.6$ & $8.3 \pm 7.3$ \\
\hline Admission to discharge & $32.4 \pm 46.9$ & $36.5 \pm 22.0$ \\
\hline \multicolumn{3}{|l|}{ Discharge setting } \\
\hline Home & $87(52.4)$ & $22(40.7)$ \\
\hline Secondary rehabilitation hospital & $67(40.4)$ & $28(51.9)$ \\
\hline Nursing home & $12(7.2)$ & $4(7.4)$ \\
\hline
\end{tabular}

Values are presented as mean \pm standard deviation or numbe (\%). MMSE, Mini-Mental State Examination; MBI, Modified Barthel Index; BBS, Berg Balance Scale; GDS, Geriatric Depression Scale. $*$ * $\mathrm{p}<0.01$. 
Table 4. Factors influencing ambulatory function at 6-month follow-up

\begin{tabular}{lcccc}
\hline \hline Ambulatory function & $\Delta \mathrm{f}$ & $\beta$ & $\mathrm{R} 2$ & $\mathrm{~F}$ \\
\hline (Constant) & 1.792 & - & 0.180 & 15.470 \\
MMSE $^{* * * *}$ & - & 0.344 & - & - \\
BBS* $^{*}$ & - & 0.190 & - & - \\
\hline
\end{tabular}

MMSE, Mini-Mental State Examination; BBS, Berg Balance Scale. *p $<0.05$. *** $<<0.001$.

bulatory function after hip fracture are summarized in Table 4. We used the backward elimination method with 7 independent variables (age, sex, bone mineral density, MMSE, BBS, premorbid ambulatory function, and length of hospital stay). The highest proportion of explained variance in ambulatory function after hip fracture was seen for the model that included MMSE and BBS $\left(R^{2}=0.180\right)$. The model factors most predictive of recovery were MMSE $(\beta=0.344, p<0.001)$ and $\operatorname{BBS}(\beta=0.190, p=0.023)$.

\section{DISCUSSION}

Only $72.3 \%$ of hip fracture patients could walk with or without assistance (independent ambulation: 1.8\%; assisted ambulation: $70.5 \%$ ) when they were transferred to rehabilitation medicine, but $86.8 \%$ could walk (independent ambulation: 70.9\%; assisted ambulation: 15.9\%) at 6 months after surgery.

In our study, $75.5 \%$ of patients involved in the ReHipFOS program achieved good ambulatory function. In other studies, hip fracture patients had difficulty achieving functional ambulatory recovery. Koot et al. ${ }^{16)}$ reported that 64 of 177 patients (36\%) had regained the level of mobility that they had before the injury at 4 months of follow-up. Of patients hospitalized for hip fractures, only $60 \%$ had recovered their pre-fracture walking ability 6 months later. ${ }^{17)}$ Kitamura et al. ${ }^{18)}$ reported that at 1-year follow-up in Japan, 67\% of hip fracture patients who underwent ambulation training after surgery, but who were not involved in a postoperative rehabilitation protocol specific to hip fracture, recovered to presurgery ambulatory status. In the present study, the prognosis for functional recovery in hip fracture patients was better than that in other studies.

According to several guidelines, patients should receive a coordinated multidisciplinary rehabilitation program after hip fracture surgery; furthermore, it is important that rehabilitation start from the time of admission. ${ }^{19,20)}$ However, current clinical pathways operating in countries other than Korea are mostly developed and maintained by orthopedic surgeons and physicians, and the focus is on reducing mortality, the length of hospital stay, and medical complications. ${ }^{21-24)}$ Furthermore, there are no proper and systematic rehabili- tation protocols for hip fracture patients in Korea. In our clinical pathway, rehabilitation is systematically organized and managed by a geriatric rehabilitation specialist, with a focus on recovery of ambulatory function. In this study, we established clinical pathways by considering various aspects of hip fracture, and these resulted in significantly improved ambulatory function at 6 months after surgery.

In previous studies, patients who were older, had cognitive impairment, or had poor ambulatory function prior to hip fracture exhibited poor functional recovery. In this study, functional recovery in hip fracture patients was associated with cognitive function and balance control upon transfer to rehabilitation. ${ }^{1625-27)}$ The mean age of our poor recovery group was not statistically different from that of the good recovery group, although it tended to be somewhat higher. Of the 220 patients, $26(11.8 \%)$ had level 2, and $14(6.4 \%)$ had level 1 preoperative ambulatory function at the initial classification. This imbalance in the number of patients in these groups may explain why significant results were not obtained for improvement relative to the premorbid state.

There are several limitations to this study. First, we initially evaluated 883 patients, but only 220 (57.4\%) visited the outpatient clinic of the Department of Rehabilitation for follow-up 6 months after surgery. The reason for the relatively low follow-up rate may be that patients with either improved or poor ambulatory function did not wish to return to the outpatient clinic. Therefore, it is possible that prognosis was over- or underestimated for these patients. Second, ambulatory function was evaluated using a 3-level grading system modified from the Functional Ambulation Category. The reason for using the 3-level scale was that the prefracture status had to be judged by referring to the premorbid function record during history-taking from the patent or caregivers. The grading criteria were very clear, and all patients enrolled in this study were assessed by one geriatric rehabilitation specialist. Third, the difference between the good and poor recovery groups was arbitrary, and the cutoff value was not clear. Finally, we did not assess ambulatory function of patients who were not involved in the ReHipFOS program after hip fracture and surgery. In this study, all patients enrolled were transferred to the Department of Rehabilitation Medicine, and all were involved in a proper rehabilitation program. Therefore, we could not directly compare the effects of ReHipFOS with the results of no treatment. For this reason, prospective comparative studies are required.

In conclusion, this study showed that a well-designed clinical pathway for hip fracture could restore ambulatory independence in most older patients. Furthermore, based on our results, the major determinants of poor ambulatory function after hip fracture include cognitive impairment and poor balance. 
Conflicts of Interest Disclosures: The researchers claim no conflicts of interest.

\section{Acknowledgments}

This research was supported by a grant of the Korea Health Technology R\&D Project through the Korea Health Industry Development Institute (KHIDI), funded by the Ministry of Health \& Welfare, Republic of Korea (grant number: HC15C1234).

\section{REFERENCES}

1. Kang HY, Yang KH, Kim YN, Moon SH, Choi WJ, Kang DR, et al. Incidence and mortality of hip fracture among the elderly population in South Korea: a population-based study using the national health insurance claims data. BMC Public Health 2010;10:230.

2. Kanis JA, Johnell O, De Laet C, Jonsson B, Oden A, Ogelsby AK. International variations in hip fracture probabilities: implications for risk assessment. J Bone Miner Res 2002;17:1237-44.

3. Haentjens P, Magaziner J, Colón-Emeric CS, Vanderschueren D, Milisen K, Velkeniers B, et al. Meta-analysis: excess mortality after hip fracture among older women and men. Ann Intern Med 2010;152:380-90.

4. Halbert J, Crotty M, Whitehead C, Cameron I, Kurrle S, Graham S, et al. Multi-disciplinary rehabilitation after hip fracture is associated with improved outcome: a systematic review. J Rehabil Med 2007;39:507-12.

5. Chevalley T, Hoffmeyer P, Bonjour JP, Rizzoli R. Early serum IGF-I response to oral protein supplements in elderly women with a recent hip fracture. Clin Nutr 2010;29:78-83.

6. Biffl WL, Smith WR, Moore EE, Gonzalez RJ, Morgan SJ, Hennessey T, et al. Evolution of a multidisciplinary clinical pathway for the management of unstable patients with pelvic fractures. Ann Surg 2001;233:843-50.

7. Koval KJ, Cooley MR. Clinical pathway after hip fracture. Disabil Rehabil 2005;27:1053-60.

8. Fisher AA, Davis MW, Rubenach SE, Sivakumaran S, Smith PN, Budge MM. Outcomes for older patients with hip fractures: the impact of orthopedic and geriatric medicine cocare. J Orthop Trauma 2006;20:172-8.

9. Adunsky A, Fleissig Y, Levenkrohn S, Arad M, Noy S. A comparative study of Mini-Mental Test, Clock Drawing task and Cognitive-FIM in evaluating functional outcome of elderly hip fracture patients. Clin Rehabil 2002;16:414-9.

10. Beaupre LA, Cinats JG, Senthilselvan A, Lier D, Jones CA, Scharfenberger A, et al. Reduced morbidity for elderly patients with a hip fracture after implementation of a perioperative evidence-based clinical pathway. Qual Saf Health Care 2006;15: 375-9.

11. Jang IY, Lee YS, Jung HW, Chang JS, Kim JJ, Kim HJ, et al. Clinical outcomes of perioperative geriatric intervention in the elderly undergoing hip fracture surgery. Ann Geriatr Med Res 2016;20:125-30.

12. Shah S, Vanclay F, Cooper B. Improving the sensitivity of the
Barthel Index for stroke rehabilitation. J Clin Epidemiol 1989; 42:703-9.

13. Kim TH, Jhoo JH, Park JH, Kim JL, Ryu SH, Moon SW, et al. Korean version of mini mental status examination for dementia screening and its' short form. Psychiatry Investig 2010;7: 102-8.

14. Kim JY, Park JH, Lee JJ, Huh Y, Lee SB, Han SK, et al. Standardization of the Korean version of the geriatric depression scale: reliability, validity, and factor structure. Psychiatry Investig 2008;5:232-8.

15. Telenius EW, Engedal K, Bergland A. Inter-rater reliability of the Berg Balance Scale, $30 \mathrm{~s}$ chair stand test and $6 \mathrm{~m}$ walking test, and construct validity of the Berg Balance Scale in nursing home residents with mild-to-moderate dementia. BMJ Open 2015;5:e008321.

16. Koot VC, Peeters PH, de Jong JR, Clevers GJ, van der Werken C. Functional results after treatment of hip fracture: a multicentre, prospective study in 215 patients. Eur J Surg 2000;166: 480-5.

17. Magaziner J, Hawkes W, Hebel JR, Zimmerman SI, Fox KM, Dolan $\mathrm{M}$, et al. Recovery from hip fracture in eight areas of function. J Gerontol A Biol Sci Med Sci 2000;55:M498-507.

18. Kitamura S, Hasegawa Y, Suzuki S, Sasaki R, Iwata H, Wingstrand $\mathrm{H}$, et al. Functional outcome after hip fracture in Japan. Clin Orthop Relat Res 1998;(348):29-36.

19. Parker MJ, Lewis SJ, Mountain J, Christie J, Currie CT. Hip fracture rehabilitation: a comparison of two centres. Injury 2002;33:7-11.

20. Mak JC, Cameron ID, March LM; National Health and Medical Research Council. Evidence-based guidelines for the management of hip fractures in older persons: an update. Med J Aust 2010;192:37-41.

21. Choong PF, Langford AK, Dowsey MM, Santamaria NM. Clinical pathway for fractured neck of femur: a prospective, controlled study. Med J Aust 2000;172:423-6.

22. Lau TW, Leung F, Siu D, Wong G, Luk KD. Geriatric hip fracture clinical pathway: the Hong Kong experience. Osteoporos Int 2010;21(Suppl 4):S627-36.

23. Olsson LE, Karlsson J, Ekman I. The integrated care pathway reduced the number of hospital days by half: a prospective comparative study of patients with acute hip fracture. J Orthop Surg Res 2006;1:3.

24. Lau TW, Fang C, Leung F. The effectiveness of a geriatric hip fracture clinical pathway in reducing hospital and rehabilitation length of stay and improving short-term mortality rates. Geriatr Orthop Surg Rehabil 2013;4:3-9.

25. Shakouri SK, Eslamian F, Azari BK, Sadeghi-Bazargani H, Sadeghpour A, Salekzamani Y. Predictors of functional improvement among patients with hip fracture at a rehabilitation ward. Pak J Biol Sci 2009;12:1516-20.

26. Marottoli RA, Berkman LF, Cooney LM Jr. Decline in physical function following hip fracture. J Am Geriatr Soc 1992;40: 861-6.

27. Press Y, Grinshpun Y, Berzak A, Friger M, Clarfield AM. The effect of co-morbidity on the rehabilitation process in elderly patients after hip fracture. Arch Gerontol Geriatr 2007;45:28194. 